\title{
MAXIMISING THE INTERFACIAL TOUGHNESS OF THIN COATINGS AND SUBSTRATE THROUGH OPTIMISATION OF DEFINED PARAMETERS
}

\author{
M.H NAZIR \& Z. KHAN \\ Bournemouth University, Faculty of Science and Technology, Sustainable Design Research Centre (SDRC). UK
}

\begin{abstract}
The influence of three parameters, i.e. interfacial roughness $\lambda$, coating thickness $h$ and impurity radius $r$ at the coating-substrate interface on interfacial toughness, has been investigated within the framework of two approaches, i.e. thermodynamics and fracture mechanics. The governing equations for both the approaches have been derived independently and then fused to form a governing law for evaluating the interfacial toughness. The analysis in this paper which considers three parameters $(\lambda, h$ and $r)$ has been divided into three setups. Each setup is used to analyse the effect of one variable parameter on interfacial toughness while keeping the other two parameters constant. Three samples for each setup were prepared considering the requirements of constant and variable parameters for each setup. Simulation techniques founded on the experimental studies have been developed during this research in order to find the optimised values of three parameters. These optimised values act as critical values (boundary point) between coating fail-safe and coating fail conditions. The experiment employed ASTM-B117 test, which is used to analyse the interfacial toughness of samples under each setup. These experiments showed excellent, quantitative agreement with the simulation trends predicted by the theoretical model. Keywords: blistering, coating failure, crack driving force, delamination, fracture mechanics, interfacial toughness, mathematical modelling, simulations, thermodynamics, strain energy release rate.
\end{abstract}

\section{INTRODUCTION}

Protective coatings tend to prevent the effects of physical and chemical attack on the substrate. However, in some circumstances this attack is promoted, rather than hindered, and this results in the debondment of coatings. This article addresses all the key parameters that play a vital role during the failure of coatings due to the debondment. The debondment of coatings from the substrate depends upon the interfacial toughness. The debonding driving force is the key element that decides the degree of interfacial toughness. Higher debonding driving force accounts for low interfacial toughness and vice versa. The debonding driving force depends on physical parameters such as interfacial roughness, coating thickness and radius of impurity at the interface. These parameters, if optimised can result in the minimum debonding driving force and therefore, reduce the probability of coating failure.

Previous analyses by Hutchinson et al. [1] of interface debondment were focused on the imperfections arising due to the thermal expansion mismatch. However, the mutual effects of interfacial roughness, coating thickness and radius of impurities at the interface have not been modelled yet due to immense complication. Also recently, models for metal coating debondment, developed by Nguyen et al. [2] and Prawoto and Dillon [3], did not address the issues related to the mutual effect of the three parameters.

This research is the continuation of existing work within Sustainable Design Research Centre (SDRC) [4-12]. This research has developed novel simulation techniques to find the critical values of debonding driving force $F$, interfacial roughness $\lambda$, coating thickness $h$ and interfacial impurity radius $\mathrm{r}$ while incorporating environmental parameters. These critical values guarantee coating safety and provide guidelines which will be used by coating manu- 
facturers to design for durability. This research utilised an experimental approach; the simulation results have been validated through the experimentation.

\section{EXPERIMENTAL}

\subsection{Samples preparation for setups $1-3$}

AISI 1010 Carbon Steel was used to prepare primer (red oxide) coated test samples with dimensions of $35 \mathrm{~mm} \times 35 \mathrm{~mm}$. The samples were categorised into three different setups on the basis of their preparation, i.e. setups 1-3.

2.1.1 Samples preparation for setup $1-$ variable interfacial roughness $\lambda$

Setup 1 was designed to analyse the effect of various roughness values $\lambda$ of the interface on the debondment driving force $F$ while keeping other two parameters constant. Three samples were prepared with interfacial roughness $(\lambda)$ of $0.0013,0.064$ and $0.21 \mu \mathrm{m}$ as shown in column 1 of Table 1 . All the samples had a constant coating thickness of $16 \mu \mathrm{m}$ and interfacial impurity radius $3.1 \mu \mathrm{m}$. The variable parameter, i.e. interfacial roughness $(\lambda)$, is highlighted as black in column 1 of Table 1 . The impurities comprise $\mathrm{NaCl}$ crystals, which were deposited on the samples before the application of coatings.

\subsubsection{Samples preparation for setup 2 - variable coating thickness $h$}

Setup 2 was designed to analyse the effect of various coating thickness $h$ values on the debondment driving force $\mathrm{F}$ while keeping other two parameters constant. Three samples were prepared with coating thicknesses (h) of 10.8, 18.2 and $43.6 \mu \mathrm{m}$ as shown in column 2 of Table 1. All the samples had a constant interfacial roughness of $0.14 \mu \mathrm{m}$ and interfacial impurity radius $3.1 \mu \mathrm{m}$. The variable parameter, i.e. coating thickness (h), is highlighted as black in column 2 of Table 1.

\subsubsection{Samples preparation for setup 3 - variable interfacial impurity radius $r$}

Setup 3 was designed to analyse the effect of interfacial impurity radii $r$ values on the debondment driving force $\mathrm{F}$ while keeping other two parameters constant. Three samples were prepared with an interfacial impurity radii (r) of 0.9, 130 and $190 \mu \mathrm{m}$ as shown in column 3 of Table 1 . All the samples had a constant interfacial roughness of $0.14 \mu \mathrm{m}$ and coating thickness $16 \mu \mathrm{m}$. The variable parameter, i.e. interfacial impurity (r), is highlighted as black in column 3 of Table 1 .

In setup 1, the debonding driving force $F$ increases with the decrease in interface roughness; therefore, sample with largest interfacial roughness $0.21 \mu \mathrm{m}$ was categorised 'safe' while the sample with smallest interface roughness $0.0013 \mu \mathrm{m}$ was categorised 'fail' as shown in column 1 of Table 1 . In setup 2, the debonding driving force $F$ increases with the decrease in coating thickness $\mathrm{h}$; therefore, sample with the largest coating thickness of $43.6 \mu \mathrm{m}$ was categorised 'safe' while the sample with the smallest coating thickness of $10.8 \mu \mathrm{m}$ was categorised 'fail' as shown in column 2. Contrary to setups 1 and 2, in setup 3, the debonding driving force $\mathrm{F}$ decreases with the decrease in the radius of interfacial impurity r; therefore, sample with smallest interfacial impurity radius $0.9 \mu \mathrm{m}$ was categorised 'safe' while the sample with largest interfacial impurity radius of $190 \mu \mathrm{m}$ was categorised 'fail' as shown in column 3. 


\subsection{Experimental observations}

Debonding driving force $\mathrm{F}$ for all the samples was measured by using an artificial method of debondment, i.e. Vickers indentation. 'Three' conditions have been defined based on the values of debonding driving force F, i.e. safe, critical and fail conditions. The 'critical condition' indicates that the samples in 'critical' condition exhibit incipient failure. The critical value of debondment driving force $\mathrm{F}_{\mathrm{c}}$ for samples is measured by averaging the values of $\mathrm{F}$ for each sample as $\mathrm{F}_{\mathrm{c}}=\left(\mathrm{F}_{\mathrm{c} 1}+\mathrm{F}_{\mathrm{c} 2}+\mathrm{F}_{\mathrm{c} 3}\right) / 3$ under setups 1-3. This average value of critical debonding driving force $F_{c}$ is equal to $0.0014 \mathrm{GPa}-\mu \mathrm{m}$. The values of variable parameters $(\lambda, h, r)$ corresponding to $F_{c}$ are treated as the critical values indicated as: $\lambda_{c}=0.064 \mu \mathrm{m}, h_{c}=18.2 \mu \mathrm{m}$ and $r_{c}=130 \mu \mathrm{m}$. These are also highlighted as 'yellow' in 'critical' condition in Table 1 .

To analyse the failure resistance of coated samples under real conditions, all the samples from each setup (1-3) were now subjected to ASTM B117 environmental test [13] at the same time. Post experimental analyses showed that the samples with maximum interface roughness $\lambda(=0.21 \mu \mathrm{m})$, maximum thickness $\mathrm{h}(=43.6 \mu \mathrm{m})$ and minimum interfacial impurity radius $r(=0.9 \mu \mathrm{m})$ exhibit maximum resistance to coating failure as shown in the category of 'safe condition' in Table 1 . This means that the samples in this category exhibit less debonding driving force $\mathrm{F}$, which has already been calculated using indentation and is shown in Fig. 1. This category is shown by 'Green highlight', which shows safe condition. Contrary to this, the samples with minimum interface roughness $\lambda(=0.013 \mu \mathrm{m})$, maximum thickness $\mathrm{h}$ $(=10.8 \mu \mathrm{m})$ and maximum interfacial impurity radius $\mathrm{r}(=190 \mu \mathrm{m})$ showed minimum resistance to coating failure as shown in the category of 'fail condition' in Table 1. This means that the samples in this category exhibit very high debonding driving force $\mathrm{F}$, which is also shown in Fig. 1. This category is shown by 'Red highlight'.

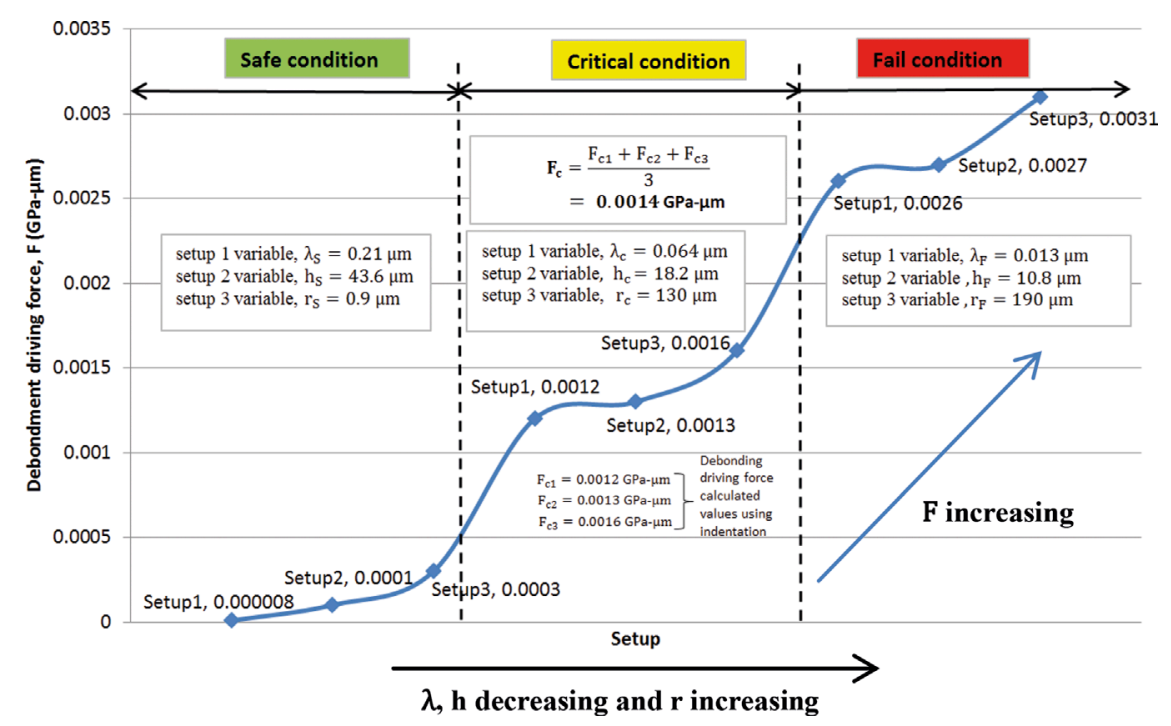

Figure 1: Categorisation of samples based on the experimental values of the debonding driving force $\mathrm{F}$. 
Table 1: Pictures of samples taken after the exposure of samples to ASTM-B117 environmental test for $150 \mathrm{~h}$.

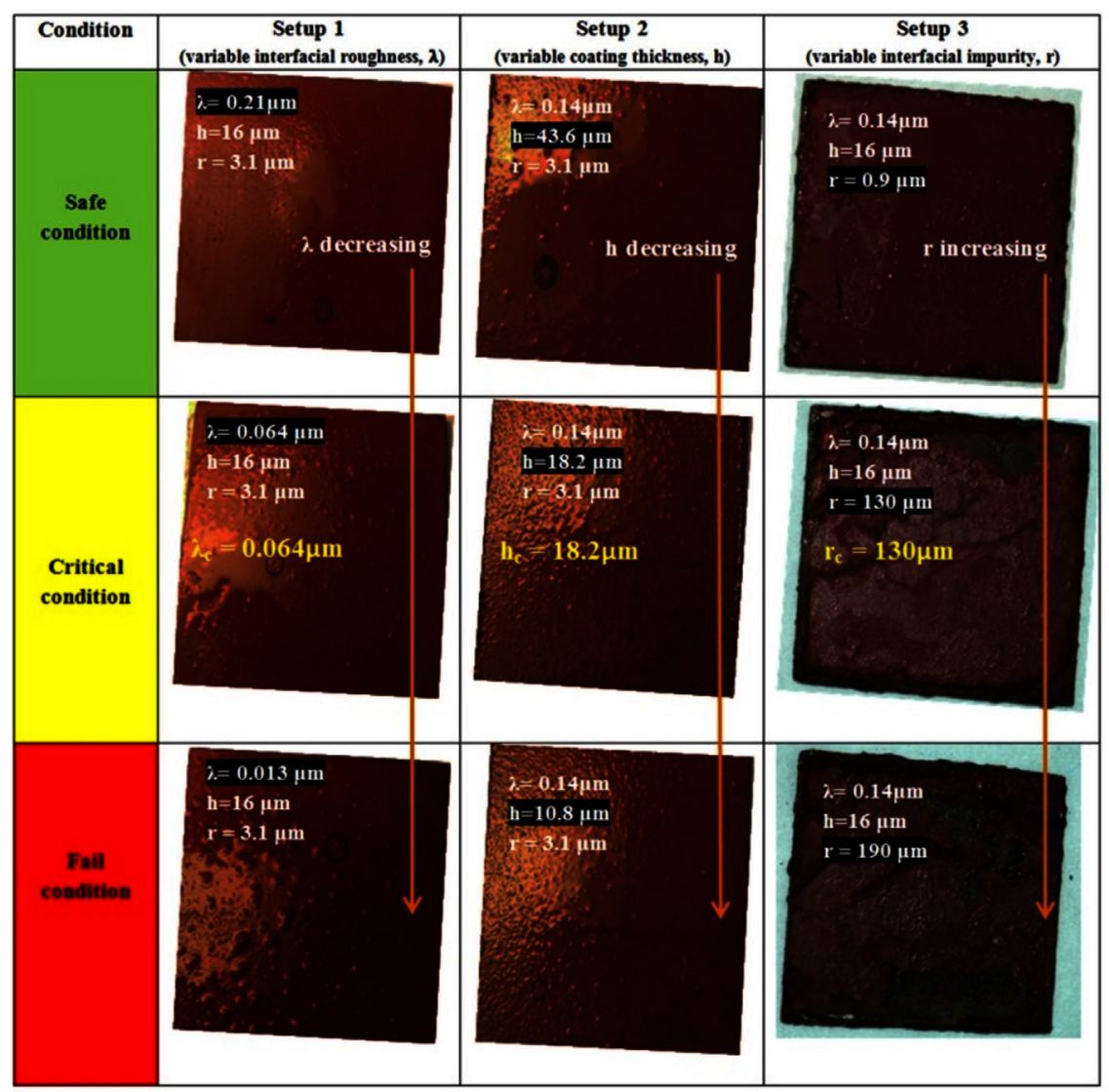

The category 'critical condition' is shown by 'Yellow highlight' in Table 1. The samples with moderate interface roughness $(=0.064 \mu \mathrm{m})$, moderate thickness $\mathrm{h}(=18.2 \mu \mathrm{m})$ and moderate interfacial impurity radius $r(=130 \mu \mathrm{m})$ exhibit a threshold or critical point for the coating failure. This means that the samples in this category have debonding driving force $\mathrm{F}$ close to the critical value of debonding driving force $F_{c}$. This critical value $F_{c}$ is the incipient requirement for the debondment initiation or failure.

\section{MATHEMATICAL FORMULATION}

The equations have been developed to analyse the failure of coatings when the samples are subjected to salt spray test such as ASTM-B117. Consider a primer-coated steel sample having interfacial roughness $\lambda$ and thickness of coating $h$ and interfacial impurity having radius $r$. The surface of coated sample is exposed to diffusing substance $\mathrm{k}(\mathrm{NaCl})$, which maintains a concentration $c_{k}$ over the coating surface as shown in Fig. 2. 


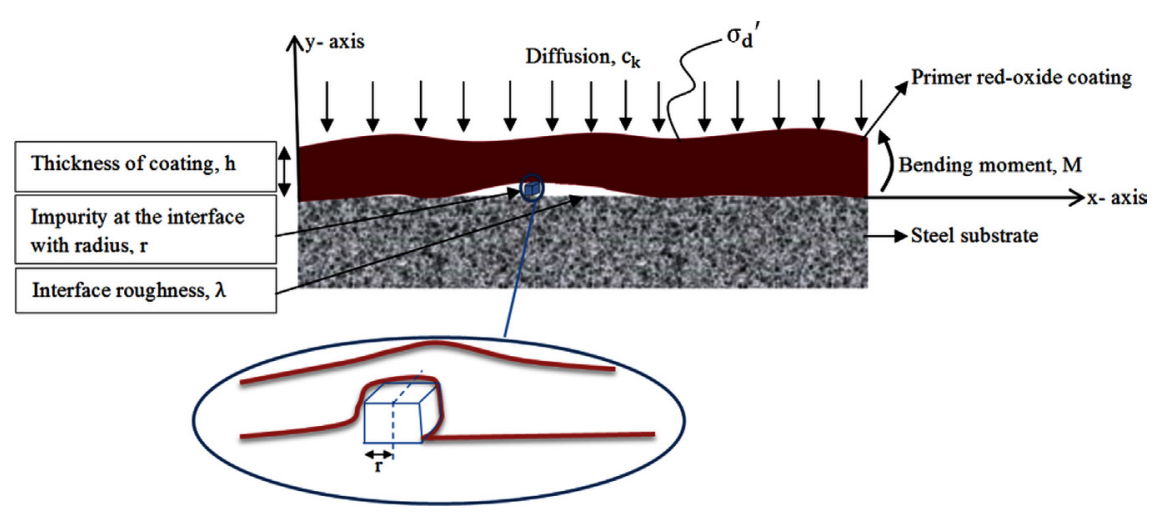

Figure 2: Primer-coated steel sample with three parameters $\lambda$, $\mathrm{h}$ and $\mathrm{r}$. The rate of change of diffusion-induced stress of coating with respect to time $\sigma_{d}{ }^{\prime}=\frac{\partial \sigma_{d}}{\partial t}$
is given as [9],

$$
\sigma_{d}^{\prime}=\frac{\partial \sigma_{d}}{\partial t}=\frac{\left(R T+E_{1} c_{k} \bar{V}_{k}^{2}\right)\left(\frac{\partial^{2} c_{k}}{\partial x^{2}}\right)+E_{1}{\overline{V_{k}}}^{2}\left(\frac{\partial c_{k}}{\partial x}\right)^{2}-\frac{R T}{D_{k_{o}}}\left(\frac{\partial c_{k}}{\partial t}\right)}{\overline{V_{k}}\left(\frac{\partial c_{k}}{\partial x}\right)}
$$

where $c_{k}$ is the mole fraction and can be treated as being equal to concentration for the infinitely diluted solid solution; $\mathrm{D}_{\mathrm{k}_{\mathrm{o}}}$ is the diffusion coefficient of substance $\mathrm{k}(\mathrm{NaCl}) ; \overline{\mathrm{V}_{\mathrm{k}}}$ is the partial molar volume of diffusing substances $\mathrm{k}(\mathrm{NaCl})$, which is dependent on molar volume of solution $\mathrm{V}_{\mathrm{m}}$ and moles of substance $\mathrm{k}$ in vapour inside chamber $\mathrm{n}_{\mathrm{k}}$; $\mathrm{E}_{1}$ is the Young modulus of the coating, $\mathrm{R}$ is the ideal gas constant and $\mathrm{T}$ is the absolute temperature inside the chamber.

The terms $c_{k}$ and $\bar{V}_{k}$ in eqn (1) can be expressed as

$$
\begin{gathered}
\mathrm{c}_{\mathrm{k}}=\frac{\mathrm{n}_{\mathrm{H}_{2} \mathrm{O}}}{\mathrm{n}_{\mathrm{H}_{2} \mathrm{O}}+\mathrm{n}_{\mathrm{k}}}=\frac{18\left[\frac{\mathrm{p}_{\mathrm{H}_{2} \mathrm{O}} \mathrm{V}_{\mathrm{r}}}{\mathrm{RT}}\right]}{18\left[\frac{\mathrm{p}_{\mathrm{H}_{2} \mathrm{O}} \mathrm{V}_{\mathrm{r}}}{\mathrm{RT}}\right]+\mathrm{n}_{\mathrm{k}}} \\
\overline{\mathrm{V}_{\mathrm{k}}}=\left(\frac{\partial \mathrm{V}_{\mathrm{m}_{\mathrm{k}}}}{\partial \mathrm{n}_{\mathrm{k}}}\right)_{\mathrm{T}, \mathrm{P}, \mathrm{n}_{\mathrm{k}}}=\left(\frac{\left.\mathrm{V}_{\mathrm{m}_{\mathrm{k}_{2}}-\mathrm{V}_{\mathrm{m}_{\mathrm{k}_{1}}}}^{\mathrm{n}_{\mathrm{k}_{2}-} \mathrm{n}_{\mathrm{k}_{2}}}\right)_{\mathrm{T}, \mathrm{P}, \mathrm{n}_{\mathrm{k} \neq \mathrm{i}}}}{\mathrm{m}_{\mathrm{m}_{\mathrm{k}_{2}}}=\frac{\mathrm{m}_{2}}{\rho_{\mathrm{k}_{2}}}, \mathrm{~V}_{\mathrm{m}_{\mathrm{k}_{1}}}=\frac{\mathrm{m}_{\mathrm{k}_{1}}}{\rho_{\mathrm{k}_{1}}}}\right.
\end{gathered}
$$


In eqn (1a), $\mathrm{p}_{\mathrm{H}_{2} \mathrm{O}}=610.78 \mathrm{e}^{\frac{17.269 \times \mathrm{T}_{\mathrm{d}}}{237.5+\mathrm{T}_{\mathrm{d}}}}$ is the vapour partial pressure of pure water dependent upon dew-point temperature $T_{d}$ inside the chamber; $V_{r}$ is the volume $\left(\mathrm{mm}^{3}\right)$ of test chamber in which sample is exposed. $\overline{V_{k}}$ in eqn (1) can be found by using Euler's first theorem for homogeneous functions as given in eqn (1b). Consider a 1 mol change in concentration $n_{k}$ from $n_{k_{1}}$ to $\mathrm{n}_{\mathrm{k}_{2}}$ keeping temperature $\mathrm{T}$ and pressure $\mathrm{P}$ constant. For such a case, $\mathrm{V}_{\mathrm{m}_{\mathrm{k} 2}}-\mathrm{V}_{\mathrm{m}_{\mathrm{k} 1}}$ is the change in molar volume associated with per molar change in concentration of a substance $k$ from $n_{k_{1}}$ to $\mathrm{n}_{\mathrm{k}_{2}}$. The molar volumes at two different stages of change are calculated using a well-known mass density relation in eqn (1c), where $\mathrm{m}_{\mathrm{k}_{2}}, \mathrm{~m}_{\mathrm{k}_{1}}$ and $\rho_{\mathrm{k}_{2}}, \rho_{\mathrm{k}_{1}}$ denote the molar masses and mass densities, respectively, at two different stages of change.

The debonding index $\prod$, which is the function of $\mathrm{h}$ and $\mathrm{r}$ can be written as

$$
\Pi(h, r)=\frac{\sigma_{d}^{\prime}}{\sigma_{c}}=\frac{\left(1-v_{1}^{2}\right)}{E_{1}}\left(\sigma_{d}^{\prime}\right)\left(\frac{r}{h}\right)^{2}
$$

where $\sigma_{\mathrm{d}}^{\prime}$ is the constant in current scenario because $\sigma_{\mathrm{d}}^{\prime}$ depends on environmental parameters such as absolute temperature $\mathrm{T}$ and moles of salt in vapour $\mathrm{n}_{\mathrm{k}}$. These parameters are kept constant in ASTM B117 environmental test. The values of $\mathrm{T}$ and $\mathrm{n}_{\mathrm{k}}$ are $\mathrm{T}=35^{\circ} \mathrm{C}$ ( or $308 \mathrm{~K}$ ) and $n_{k}$ is the molar conversion of $5 \% \mathrm{NaCl}$ solution. The term $\sigma_{c}$ is the critical stress when the coating just begins to debond from the substrate. Upon substituting eqn (1) in eqn (2), the debonding index becomes

$$
\begin{aligned}
\Pi(\mathrm{h}, \mathrm{r})=\frac{\sigma_{\mathrm{d}}^{\prime}}{\sigma_{\mathrm{c}}}=\left(\frac{1-\mathrm{v}_{1}^{2}}{\mathrm{E}_{1}}\right) \\
\frac{\left(\mathrm{RT}+\mathrm{E}_{1} \mathrm{c}_{\mathrm{k}} \overline{\mathrm{V}}_{\mathrm{k}}^{2}\right)\left(\frac{\partial^{2} \mathrm{c}_{\mathrm{k}}}{\partial \mathrm{x}^{2}}\right)+\mathrm{E}_{1}{\overline{\mathrm{V}_{\mathrm{k}}}}^{2}\left(\frac{\partial \mathrm{c}_{\mathrm{k}}}{\partial \mathrm{x}}\right)^{2}-\frac{\mathrm{RT}}{\mathrm{D}_{\mathrm{k}_{\mathrm{o}}}}\left(\frac{\partial \mathrm{c}_{\mathrm{k}}}{\partial \mathrm{t}}\right)}{\overline{\mathrm{V}_{\mathrm{k}}}\left(\frac{\partial \mathrm{c}_{\mathrm{k}}}{\partial \mathrm{x}}\right)}\left(\frac{\mathrm{r}}{\mathrm{h}}\right)^{2}
\end{aligned}
$$

where $\mathrm{r}$ is the radius of initial impurity defect at the interface and $\mathrm{h}$ is the coating thickness.

It is well known that strain energy release rate $G$ is a measure of the driving force for debondment propagation. For the interface defect problem, G, Hutchinson's equation [14], which was purely based on fracture mechanics parameters, can now be modified in terms of thermodynamics and fracture mechanics parameters by incorporating eqn (3) as

$$
\mathrm{G}(\mathrm{h}, \mathrm{r})=\frac{6\left(1-\mathrm{v}_{1}^{2}\right)}{\mathrm{E}_{1} \mathrm{~h}^{3}} \mathrm{M}^{2}\left[1+\left(0.5 \sqrt{\mathrm{c}_{1}(\Pi-1)}\right)^{2}\right]
$$

where $\mathrm{M}$ in eqn (4) is the bending moment that results in the edge-crack, which separates the coating from the substrate; $c_{1}=\frac{\left(1+v_{1}\right)^{2}}{\left(1+v_{1}\right)+\left(1-v_{1}^{2}\right)}$ is the constant, which depends upon 
Poisson's ratio of the coating. Mode-dependent strain energy release rate $G(h, r)$ due to debonding can be adjusted using mode-mix function $\mathrm{j}(\lambda, \mathrm{h}, \mathrm{r})$. Debonding propagation depends on mode-adjusted debondment driving force $F(\lambda, h, r)$ and is given as follows:

$$
F(\lambda, h, r)=\frac{G(h, r)}{j(\lambda, h, r)}
$$

where $\mathrm{j}(\lambda, \mathrm{h}, \mathrm{r})$ in eqn (6) can be written as

$$
\begin{gathered}
\mathrm{j}(\lambda, \mathrm{h}, \mathrm{r})=1+\tan ^{2}\left[\left(1-\frac{\Gamma_{\mathrm{IC}}}{\mathrm{E}_{1} \lambda}\right) \psi\right] \\
\psi=\frac{\mathrm{K}_{\mathrm{II}}}{\mathrm{K}_{\mathrm{I}}}=\tan ^{-1}\left(\frac{\cos \omega+\left[0.2486\left(1+\mathrm{v}_{1}\right)\left(\sqrt{\frac{1}{\mathrm{c}_{2}}(\Pi-1)}\right)\right] \sin \omega}{-\sin \omega+\left[0.2486\left(1+\mathrm{v}_{1}\right)\left(\sqrt{\frac{1}{\mathrm{c}_{2}}(\Pi-1)}\right)\right] \cos \omega}\right)
\end{gathered}
$$

In eqn (6), $\psi$ is the ratio of mode II to mode I stress intensity factors; $\Gamma_{I C}=G_{c}(h, r)$ is the mode 1 toughness and is equal to $G_{c}$, where $G_{c}$ is the incipient energy release rate at critical condition; $\mathrm{c}_{2}=0.2\left[\left(1+\mathrm{v}_{1}\right)+\left(1-\mathrm{v}_{1}^{2}\right)\right]$ and $\omega$ is dependent on elastic mismatch parameter $\alpha=\left(\overline{\mathrm{E}_{1}}-\overline{\mathrm{E}_{2}}\right) /\left(\overline{\mathrm{E}_{1}}+\overline{\mathrm{E}_{2}}\right)$, where $\mathrm{E}_{2}$ is the elastic modulus of the substrate.

Utilising eqn (5), three conditional functions in eqns (7a)-(7c) are developed to find the critical values of parameters: interface roughness $\lambda$, coating thickness $h$ and interfacial impurity size r, respectively.

$$
\begin{aligned}
& \begin{array}{c}
\text { Setup 1 } \\
\text { Variable } \lambda
\end{array} \quad \mathrm{F}(\lambda, \mathrm{h}, \mathrm{r}) \begin{cases}<G(\mathrm{~h}, \mathrm{r}) \text { if } \lambda>\lambda_{\mathrm{c}} & \text { (Safe condition) } \\
\rightarrow G(\mathrm{~h}, \mathrm{r}) \text { if } \lambda=\lambda_{\mathrm{c}} & \text { (Critical condition) } \\
=G(\mathrm{~h}, \mathrm{r}) \text { if } \lambda<\lambda_{\mathrm{c}} & \text { (Fail condition) }\end{cases}
\end{aligned}
$$

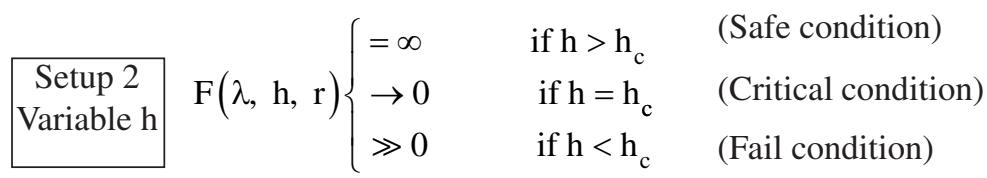

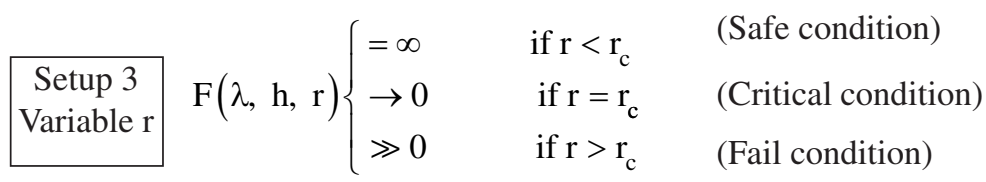

For each setups 1-3, there is one variable parameter and two constant parameters based on experimental design. Each setup accounts for three distinct conditions, i.e. safe, critical and fail as shown in eqns (7b) and (7c), respectively.

The critical condition gives the critical debondment driving force $\mathrm{F}_{\mathrm{c}}$, which is the incipient requirement of debonding. The critical debondment driving force for $\mathrm{F}_{\mathrm{c}}$ each setup exists when 
the corresponding value of variable parameter is equal to its critical value. For the case of setups 2 and 3 , when variable parameters are $\mathrm{h}$ and $\mathrm{r}$, respectively, the critical value $\mathrm{F}_{\mathrm{c}}$ is very small close to zero $(\rightarrow 0)$ as given in eqns (7b) and (7c). However, for the case of setup 1, when the variable parameter is $\lambda$, then the critical value $F_{c}$ approach $G(h, r)$ as given in eqn (7a).

Setup 1 shows that even for safe condition, i.e. $\lambda>\lambda_{c}, F$ is fully dependent on $G$ (strain energy release rate), where $G$ is a function of $h$ and $r$ as shown in eqn (4). Therefore, for very high $\mathrm{G}$ due to larger $\mathrm{r}$ and smaller $\mathrm{h}$, the debonding driving force $\mathrm{F}$ will be very high, even if the interface roughness $\lambda$ is high (greater than critical value $\lambda_{c}$ ).

\section{RESULTS AND DISCUSSION}

This section discusses the simulation results obtained by utilising the expressions for debondment driving force $\mathrm{F}$ in eqns $(7 \mathrm{a})-(7 \mathrm{c})$. This section also compares the simulation results with the experimental results to validate the accuracy of theoretical results. The numerical simulations have been designed using the finite difference method. The parameters in Table 2 were used during the numerical simulation.

\subsection{Setup 1: Constant $\mathrm{h}$ and $\mathrm{r}$ with variable $\lambda$}

The simulation results for setup 1 in Fig. 3 with variable parameter as interface roughness $\lambda$ show that for the case when $\lambda>\lambda_{\mathrm{c}}$, the debonding driving force $\mathrm{F}$ is very small compared

Table 2: Parameters used in analysis.

\begin{tabular}{cccc}
\hline $\mathrm{T}(\mathrm{K})$ & $\mathrm{T}_{\mathrm{d}}(\mathrm{K})$ & $\mathrm{V}_{\mathrm{r}}\left(\mathrm{mm}^{3}\right)$ & $\rho_{\mathrm{k}_{\mathrm{NaCl}}}\left(\mathrm{g} / \mathrm{mm}^{3}\right)$ \\
\hline 308 & 285 & $5 \times 10^{8}$ & 0.00216 \\
\hline
\end{tabular}

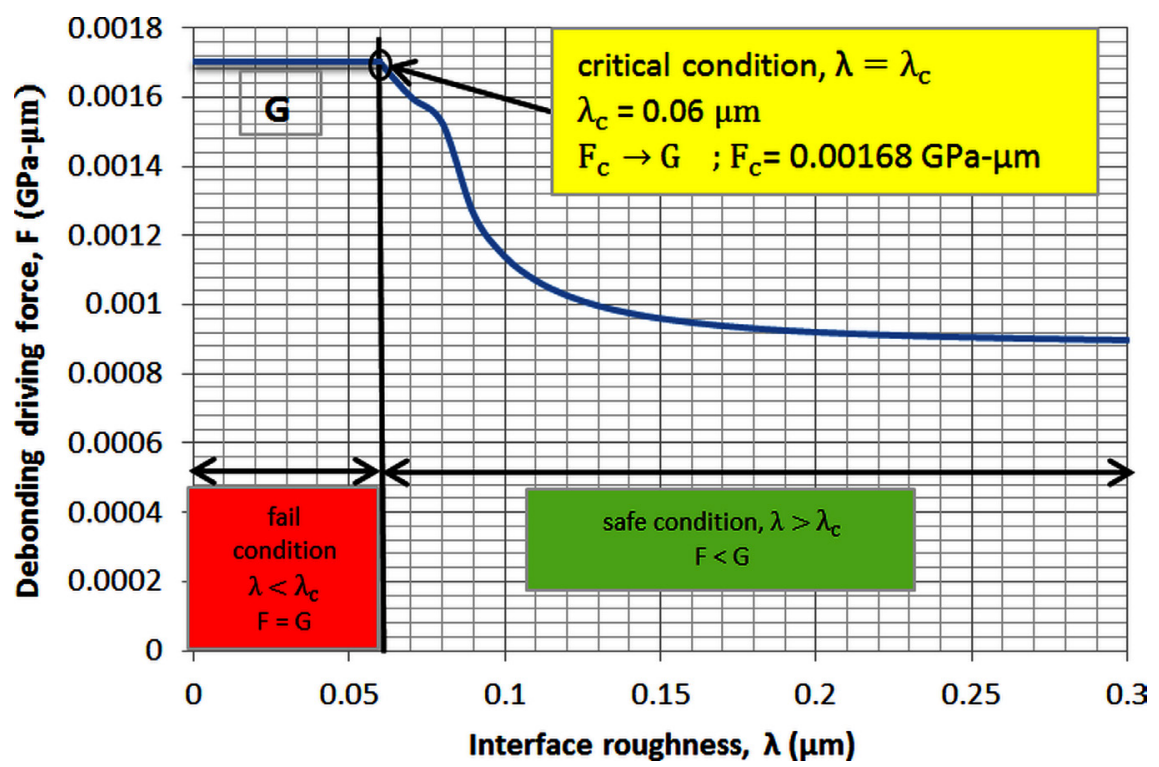

Figure 3: Debonding driving force as a function of interface roughness $\lambda$. 
Table 3: Experimental and simulation results for setup 1 and their $\%$ age reliability.

\begin{tabular}{llll}
\hline & Experimental results & Simulation results & $\%$ Reliability \\
\hline$\lambda_{\mathrm{c}}(\mu \mathrm{m})$ & 0.064 & 0.06 & 93.5 \\
$\mathrm{~F}_{\mathrm{c}}(\mathrm{GPa}-\mu \mathrm{m})$ & 0.0014 & 0.00168 & 81.82 \\
\hline
\end{tabular}

with strain energy release rate $\mathrm{G}$, i.e. $\mathrm{F}<\mathrm{G}$. This makes the safe condition. Contrary to this when $\lambda>\lambda_{c}$, then the debonding driving force is equal to $G$, i.e. $F=G$. This makes the fail condition. The critical value of $\mathrm{F}$, which is $\mathrm{F}_{\mathrm{c}}$, is found when $\lambda=\lambda_{\mathrm{c}}$. For this case, F approach strain energy release rate $G$, i.e. $F \rightarrow G$.

Simulation results show that the debonding driving force $F$ decreases with an increase in interface roughness $\lambda$ as shown in Fig. 3 . This decrease in $F$ with an increase in $\lambda$ means that the interface becomes tougher with an increase in interface roughness $\lambda$. The debonding driving force $F$ becomes stable after certain value of interface roughness $\lambda$.

The critical value of interface roughness $\lambda_{c}=0.06 \mu \mathrm{m}$ and critical debonding driving force $\mathrm{F}_{\mathrm{c}}=0.00168 \mathrm{GPa}-\mu \mathrm{m}$ are found using numerical simulations based on conditional function in eqn (7a). These simulation results are compatible with the critical values, which are found using experimental analysis. These critical values found using experimental investigation are, i.e. $\lambda_{\mathrm{c}}=0.064 \mu \mathrm{m}$ and $\mathrm{F}_{\mathrm{c}}=0.0014 \mathrm{GPa}-\mu \mathrm{m}$. The $\%$ age reliability in Table 3 shows the comparison of experimental and the simulation results and also the degree of accuracy of both the results.

\subsection{Setup 2: Constant $\lambda$ and $r$ with variable $h$}

The simulation results for setup 2 in Fig. 4(a) and (b), with variable parameter as coating thickness $h$ show that when $h=h_{c}$, the debondment driving force $F$ is very small and its value approach zero, i.e. $\mathrm{F} \rightarrow 0$. It is to be noted that for primer red-oxide, the increase in $\mathrm{h}$ is accompanied by the decrease in Young's modulus of the coating $E_{1}$ as shown in Fig. 4(b). As per experimental data, the elastic modulus $\mathrm{E}_{1}$, for every $100 \%$ change in coating thickness, $\mathrm{E}_{1}$ for coating decreases by $\mathrm{k}=26.7 \%$. This percentage change in $\mathrm{E}_{1}$ is specifically recorded for primer (red-oxide) subjected to ASTM-B117 test condition, i.e. $\mathrm{T}=35^{\circ} \mathrm{C}$ and $5 \% \mathrm{NaCl}$ solution. The change in $\mathrm{E}_{1}$ effects the elastic mismatch parameter $\alpha$ and is, in turn, affecting $\omega$ and $\psi$, where $\psi$ is used to fine tune $\mathrm{j}(\lambda, \mathrm{h}, \mathrm{r})$, which is used to adjust the debonding driving force $\mathrm{F}$ as given in eqn (6).

Simulation results show that the debonding driving force $\mathrm{F}$ decreases with an increase in coating thickness $\mathrm{h}$ and increases rapidly as the coating thickness approach nanoscale. When the thickness of the coating is extremely small $\left(<<\mathrm{h}_{\mathrm{c}}\right)$ in the scale of $\mathrm{nm}$, than the debonding driving force attains $10^{4}$ order, which is very high. Usually, the coating debonds from the substrate if the thickness of the coating is $<19.8 \mu \mathrm{m}$, which is the critical thickness of the coating. In this case, the critical value of debonding driving force is about $0.0011 \mathrm{GPa}-\mu \mathrm{m}$. These values are found using numerical simulations based on conditional function in eqn (7b).

These simulation results are compatible with the critical values, which are found using experimental analysis. The critical values found using experimental investigation are $\mathrm{h}_{\mathrm{c}}=18.2 \mu \mathrm{m}$ and $\mathrm{F}_{\mathrm{c}}=0.0014 \mathrm{GPa}-\mu \mathrm{m}$. The $\%$ age reliability between experimental and simulation results is shown in Table 4. 


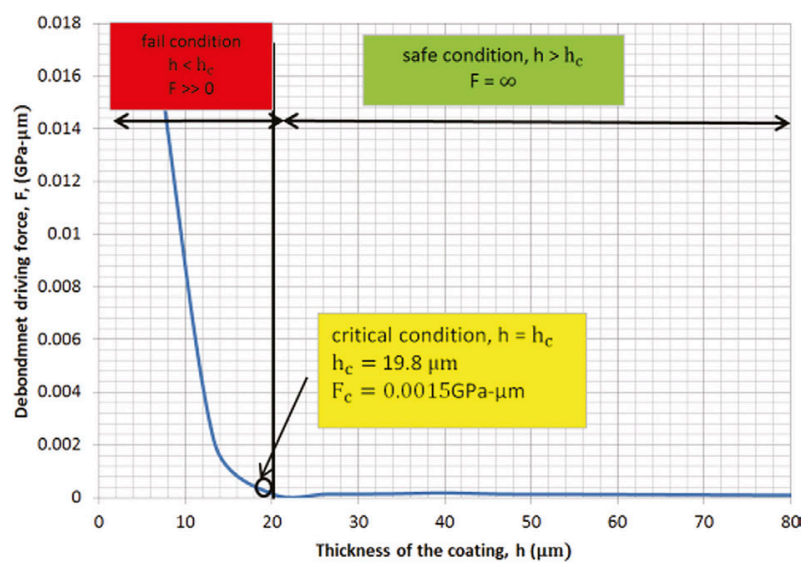

(a)

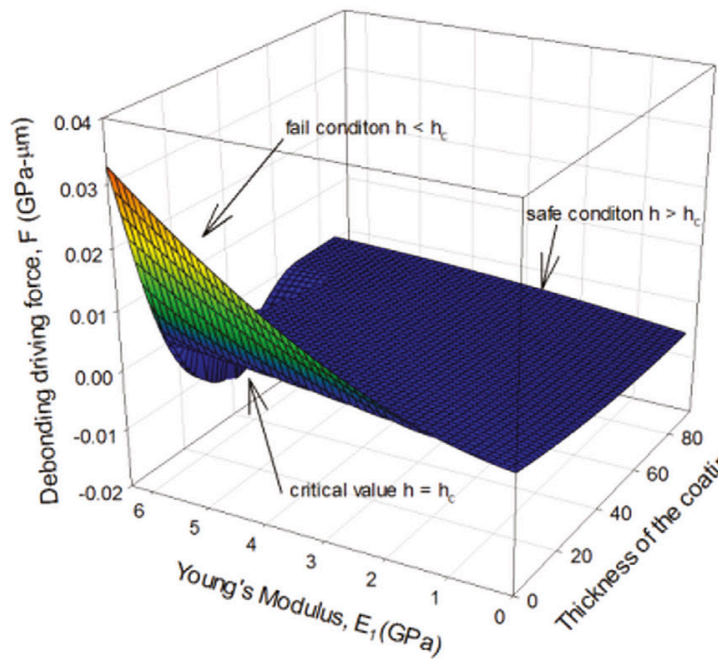

(b)

Figure 4: (a) Debonding driving force as a function of coating thickness $h$ and (b) 3D mesh for $\mathrm{F}$ as a function of coating thickness $\mathrm{h}$ and Young's modulus $\mathrm{E}_{1}$.

Table 4: Experimental and simulation results for setup 2 and their $\%$ age reliability.

\begin{tabular}{lccc}
\hline & Experimental results & Simulation results & \% Reliability \\
\hline $\mathrm{h}_{\mathrm{c}}(\mu \mathrm{m})$ & 18.2 & 19.8 & 91.5 \\
$\mathrm{~F}_{\mathrm{c}}(\mathrm{GPa}-\mu \mathrm{m})$ & 0.0014 & 0.0015 & 93.1 \\
\hline
\end{tabular}

\subsection{Setup 3: constant $\lambda$ and $h$ with variable $r$}

The simulation results for setup 3 in Fig. 5, with variable parameter as impurity radius $r$ show that for the case when $r=r_{c}$, the debondment driving force $F$ is very small and its value approach zero, i.e. $\mathrm{F} \rightarrow 0$. 


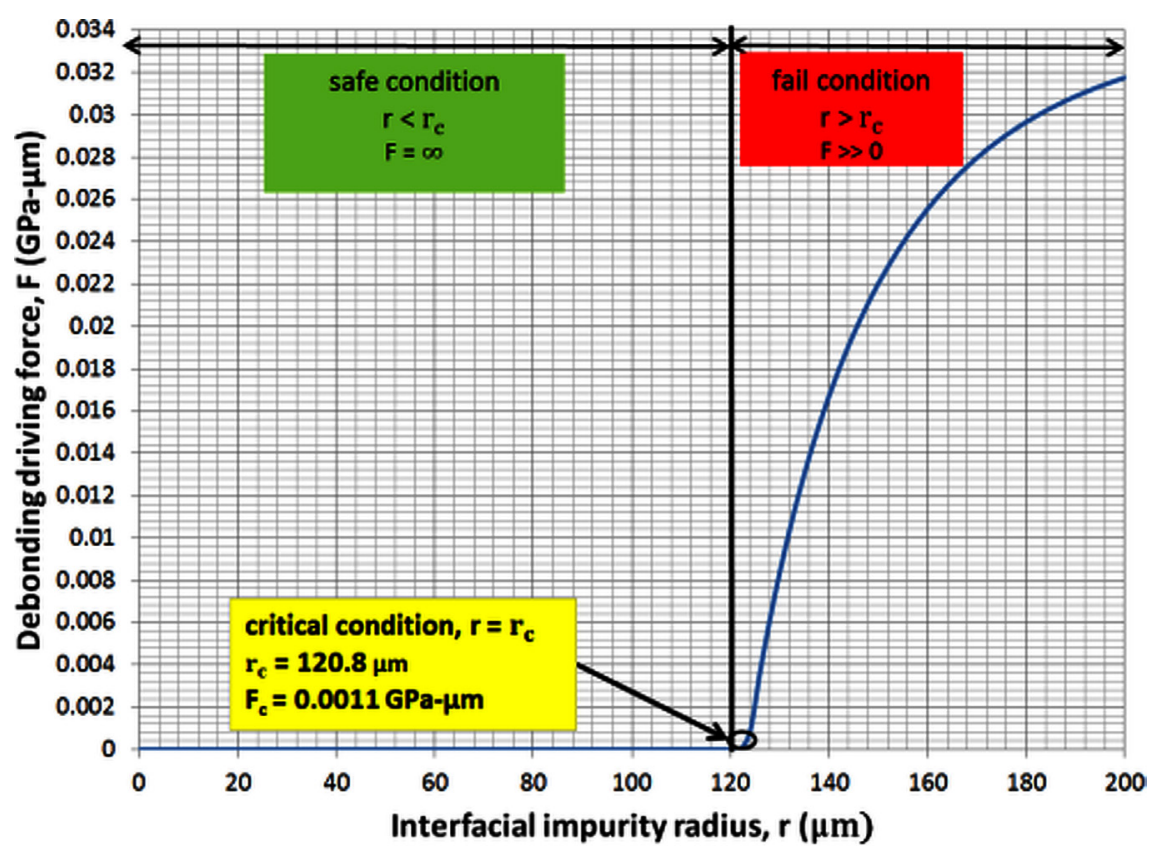

Figure 5: Debonding driving force as a function of radius of impurity at the interface $r$.

Table 5: Experimental and simulation results for setup 3 and their $\%$ age reliability.

\begin{tabular}{lccc}
\hline & Experimental results & Simulation results & $\%$ Reliability \\
\hline $\mathrm{r}_{\mathrm{c}}(\mu \mathrm{m})$ & 130 & 120.8 & 92.8 \\
$\mathrm{~F}_{\mathrm{c}}(\mathrm{GPa}-\mu \mathrm{m})$ & 0.0014 & 0.0011 & 76 \\
\hline
\end{tabular}

Simulation results show that the debonding driving force $\mathrm{F}$ decreases with the decrease in $r$ as shown in Fig. 5. This decrease in $F$ with the decrease in $r$ means that the interface becomes tougher. The critical value of debonding driving force $F_{c}$ is found by using conditional function in eqn (7c). The function returns a value close to zero $(\rightarrow 0)$, which is the condition for incipient fracture. This condition occurs if $G \rightarrow 0$ and $j(\lambda, h, r) \rightarrow 1$ in eqn (5). However, there is a large rise in $\mathrm{F}$ with the rise in radius of interfacial impurity $\mathrm{r}$ under the fail condition. The conditional function returns a fail condition, i.e. $F>G$ where $G>>0$, which also makes $F>>0$. For this case, eqn (6) always returns value of $F$ greater than $G$ and zero. This coating fail condition only occurs if $r>r_{c}$.

These simulation results are compatible with the critical values, which are found using experimental analysis. These critical values found using experimental investigation are $r_{c}=120.8 \mu \mathrm{m}$ and $\mathrm{F}_{\mathrm{c}}=0.0011 \mathrm{GPa}-\mu \mathrm{m}$. The $\%$ age reliability between experimental and simulation results is shown in Table 5. 


\section{CONCLUSIONS}

The debondment of coating from the substrate is a multidisciplinary problem. There was always a need of a multi-dimensional approach to investigate the problem of coatingsubstrate failure due to debondment. This article, in particular, addresses the solution by fostering a close collaboration between two major disciplines, i.e. material science and solid mechanics. The equations for both the disciplines have been designed independently and then fused to form a governing law to predict the failure and analyse the service life of coatings bonded to the substrate. The debonding driving force $\mathrm{F}$ is the key element responsible for the coating debondment.

The novelty in this article lies in designing a numerical model by the integration of two distinct fields (material science and solid mechanics). This approach has been utilised to design an equation for the debonding driving force F. However, there is a room for improvement in the current design by expanding the parameters and including the electrochemistry as a third discipline. By integrating the electrochemistry concepts with the solid mechanics and material science, novel equations for corrosion current density can be deigned, which can bind and correlate parameters such as the ionic concentration, stress components with corrosion current density. Extensive experimental and simulation work [7-11] is being done in our research group (SDRC Bournemouth University, UK) to come up with an optimum design, which can address all the current issues related to coating-substrate debondment.

The developed model in this research can be utilised in prognostics, which is the ability to predict the remaining useful life of a failing system [15]. In this research, the failing system is a coating-substrate system. The physical quantity to be predicted is the 'metal-coating adhesion failure'. The objective is to give the numerical model the form of software, which if integrated with real-time sensors can be used to monitor the failure of coatings on various structures, particular of historic importance. This technique can provide the ability to pre-empt expensive and catastrophic structural failures.

\section{REFERENCES}

[1] Hutchinson, J., He, M. \& Evans, A., The influence of imperfections on the nucleation and propagation of buckling driven delaminations. Journal of the Mechanics and Physics of Solids, 48, pp. 709-734, 2000. doi: http://dx.doi.org/10.1016/S0022-5096(99)00050-2

[2] Nguyen, T., Hubbard, J. \& Pommersheim, J., Unified model for the degradation of organic coatings on steel in a neutral electrolyte. Journal of Coatings Technology, 68, pp. 45-56, 1996.

[3] Prawoto, Y. \& Dillon, B., Failure analysis and life assessment of coating: the use of mixed mode stress intensity factors in coating and other surface engineering life assessment. Journal of Failure Analysis and Prevention, 12, pp. 190-197, 2012. doi: http://dx.doi.org/10.1007/s11668-011-9525-1

[4] Saeed, A., Khan, Z., Clark, M., Nel, M. \& Smith, R., Non-destructive material characterisation and material loss evaluation in large historic military vehicles. Insight-Non-Destructive Testing and Condition Monitoring, 53, pp. 382-386, 2011. doi: http://dx.doi.org/10.1784/insi.2011.53.7.382

[5] Saeed, A., Khan, Z.A., Hadfield, M. \& Davies, S., Material characterization and real-time wear evaluation of pistons and cylinder liners of the Tiger 131 Military Tank. Tribology Transactions, 56, pp. 637-644, 2013. doi: http://dx.doi.org/10.1080/10402 004.2013.771416 
[6] Saeed, A., Khan, Z.A. \& Montgomery, E., Corrosion damage analysis and material characterization of Sherman and Centaur - the historic military tanks. Materials Performance and Characterization, 2, pp. 1-16, 2013. doi: http://dx.doi.org/10.1520/ MPC20120016

[7] Nazir, M.H., Khan, Z. \& Stokes, K., Modelling of metal-coating delamination incorporating variable environmental parameters. Journal of Adhesion Science and Technology, 29(5), pp. 1-32, 2014.

[8] Nazir, M.H., Khan, Z. \& Stokes, K., Adhesive threshold predicitive modelling in the presence of interfacial impurity. Journal of Adhesion Science and Technology, 2015 (submitted).

[9] Nazir, M.H., Khan, Z.A. \& Stokes, K., Optimisation of interface roughness and coating thickness to maximise coating-substrate adhesion - a failure prediction and reliability assessment modelling. Journal of Adhesion Science and Technology, 29(14), pp. 14151445, 2015. doi: http://dx.doi.org/10.1080/01694243.2015.1026870

[10] Nazir, M.H., Khan, Z.A. \& Stokes, K., A holistic mathematical modelling and simulation for cathodic delamination mechanism - a novel and an efficient approach. Journal of Adhesion Science and Technology, 2015 (submitted).

[11] Nazir, M.H., Khan, Z.A. \& Stokes, K., A unified mathematical modelling and simulation for cathodic blistering mechanism incorporating diffusion and fracture mechanics concepts. Journal of Adhesion Science and Technology, 29(12), pp. 1-29, 2015. doi: http://dx.doi.org/10.1080/01694243.2015.1022496

[12] Khan, Z.A., Pashaei, P., Bajwa, R.S., Nazir, M.H. \& Camak, M., Fabrication and characterisation of electrodeposited and magnetron sputtered thin films. International Journal of Computational Methods \& Experimental Measurements, 2015 (submitted).

[13] Yang, F. \& Li, J.C.-M., Micro and Nano Mechanical Testing of Materials and Devices, New York, NY: Springer, 2008.

[14] Hutchinson, J., Thouless, M. \& Liniger, E., Growth and configurational stability of circular, buckling-driven film delaminations. Acta Metallurgica et Materialia, 40, pp. 295-308, 1992. doi: http://dx.doi.org/10.1016/0956-7151(92)90304-W

[15] Vachtsevanos, G., Lewis, F., Roemer, M., Hess, A. \& Wu, B., Intelligent fault diagnosis and prognosis for engineering systems. Usa 454p Isbn, 13, pp. 978-1000, 2006. doi: http://dx.doi.org/10.1002/97804701178423 